\title{
Article \\ Efficacy of Vitamin D Supplements in Prevention of Acute Respiratory Infection: A Meta-Analysis for Randomized Controlled Trials
}

\author{
Hae-Eun Cho ${ }^{1}$, Seung-Kwon Myung ${ }^{2,3,4, *}$ and Herim Cho ${ }^{1}$ \\ 1 Department of Medicine, College of Medicine, Ewha Womans University, Seoul 07804, Korea; \\ puerile97@naver.com (H.-E.C.); herimcho0725@gmail.com (H.C.) \\ 2 Department of Cancer Biomedical Science, National Cancer Center Graduate School of Cancer Science and \\ Policy, Goyang 10408, Korea \\ 3 Cancer Epidemiology Branch, Division of Cancer Data Science, National Cancer Center Research Institute, \\ Goyang 10408, Korea \\ 4 Department of Family Medicine and Center for Cancer Prevention and Detection, National Cancer Center \\ Hospital, Goyang 10408, Korea \\ * Correspondence: msk@ncc.re.kr; Tel.: +82-31-920-0479
}

check for updates

Citation: Cho, H.-E.; Myung, S.-K.; Cho, H. Efficacy of Vitamin D Supplements in Prevention of Acute Respiratory Infection: A MetaAnalysis for Randomized Controlled Trials. Nutrients 2022, 14, 818 https://doi.org/10.3390/ nu14040818

Academic Editors: Federica I. Wolf and Raffaele Antonelli Incalzi

Received: 20 January 2022

Accepted: 9 February 2022

Published: 15 February 2022

Publisher's Note: MDPI stays neutral with regard to jurisdictional claims in published maps and institutional affiliations.

Copyright: (C) 2022 by the authors. Licensee MDPI, Basel, Switzerland. This article is an open access article distributed under the terms and conditions of the Creative Commons Attribution (CC BY) license (https:// creativecommons.org/licenses/by/ $4.0 /)$.

\begin{abstract}
Background: Previous systematic reviews and meta-analyses of randomized controlled trials (RCTs) have reported inconsistent results regarding the efficacy of vitamin D supplements in the prevention of acute respiratory infections (ARIs). Methods: We investigated these efficacy results by using a meta-analysis of RCTs. We searched PubMed, EMBASE, and the Cochrane Library in June 2021. Results: Out of 390 trials searched from the database, a total of 30 RCTs involving 30,263 participants were included in the final analysis. In the meta-analysis of all the trials, vitamin D supplementation showed no significant effect in the prevention of ARIs (relative risk (RR) 0.96, $95 \%$ confidence interval (CI) $0.91-1.01, \mathrm{I}^{2}=59.0 \%, n=30$ ). In the subgroup meta-analysis, vitamin D supplementation was effective in daily supplementation (RR $0.83,95 \%$ CI, 0.73-0.95, $\mathrm{I}^{2}=69.1 \%$, $n=15$ ) and short-term supplementation (RR 0.83, 95\% CI, $0.71-0.97, \mathrm{I}^{2}=66.8 \%, n=13$ ). However, such beneficial effects disappeared in the subgroup meta-analysis of high-quality studies (RR 0.89 , $95 \%$ CI $, 0.78-1.02, \mathrm{I}^{2}=67.0 \%, n=10$ assessed by the Jadad scale; RR 0.87, 95\% CI, $0.66-1.15, \mathrm{I}^{2}=51.0 \%$, $n=4$ assessed by the Cochrane's risk of bias tool). Additionally, publication bias was observed. Conclusions: The current meta-analysis found that vitamin D supplementation has no clinical effect in the prevention of ARIs.
\end{abstract}

Keywords: vitamin D supplements; acute respiratory infections; randomized controlled trial; meta-analysis

\section{Introduction}

Acute respiratory infection (ARI) is classified into an upper respiratory tract infection (URI) and a lower respiratory tract infection (LRI). URIs include common cold (nasopharyngitis), sinusitis, pharyngitis, laryngitis, and laryngotracheitis [1]. The common cold, as a frequent cause of URIs, is caused by viral infections such as rhinovirus, coronavirus, influenza virus A/B/C, respiratory syncytial virus, parainfluenza virus, and adenovirus [1]. URIs are a common disease, of which adults experience 2-4 episodes a year on average, and children 7-12 episodes [2]. LRIs are mostly caused by viruses such as the influenza virus and respiratory syncytial virus. Moreover, they are caused by bacterial infections such as $S$. aureus, S. pneumoniae, and H. influenza, tuberculosis infections, fungal infections, and parasite infections [3]. LRIs have been the fifth leading cause of death and responsibility for mortality in adults older than 70 years worldwide since 1990, accounting for up to 94.6 per 1000 global deaths [4]. 
The U.S. Centers for Disease Control and Prevention (CDC) recommends several useful preventive measures for ARIs such as avoiding close contact with a sick person and practicing hygiene: regular handwashing, covering nose and mouth, or using tissues to contain respiratory droplets or secretions. Vaccination against influenza, pneumococcus, and tuberculosis is also being used for the primary prevention of ARIs. Additionally, although oral zinc, vitamin C supplements, vitamin D supplements, ginseng, and probiotics have been suggested to have a preventive effect on the development of ARIs in some studies, this remains inconclusive [5,6], whereas a meta-analysis of six randomized controlled trials (RCTs) reported that lactoferrin supplements, as one of the key immunomodulatory substances, had efficacy in reducing the risk of RTIs [7].

In the meantime, it has been reported that vitamin $\mathrm{D}$, which has an important role in calcium and bone homeostasis, affects the immune system [8]. From the previous laboratory studies, $1,25(\mathrm{OH}) 2 \mathrm{D}$, which is an active form of vitamin $\mathrm{D}$, is related to innate and adaptive immunity: it enhances the antibacterial responses of innate immune cells and inhibits $\mathrm{T}$ cell proliferation and cytokine excretion from helper T cells, and downregulates chronic T cell-mediated reactions [8-10]. Additionally, an animal study showed that vitamin D could suppress influenza virus replication and inflammation in a mouse model [11]. On the contrary, vitamin D deficiency, which is also associated with nonalcoholic fatty liver disease, obesity, or metabolic syndrome, is known to be linked to an increased risk of infections [12].

Furthermore, several RCTs have reported the preventive effects of vitamin D supplements on the incidence of ARIs [13-23], while others have reported no effect [24-42]. Several meta-analyses reported whether there are beneficial effects $[6,43-46]$. However, Pham et al. [44] and Martineau et al.'s [45] meta-analyses investigated the association between $25(\mathrm{OH}) \mathrm{D}$ concentration (not supplementation) and the risk of ARIs but lacked the information about actual doses and regimens for vitamin D supplementation.

The current study aimed to investigate whether vitamin D supplementation reduces the risk of ARIs by using a meta-analysis of RCTs. We conducted various subgroup metaanalyses by important factors such as the duration of supplementation, vitamin dosage, and number of study participants.

\section{Methods and Materials}

\subsection{Data Sources and Search}

We searched the Cochrane Library, Embase, and PubMed in order to retrieve articles about the effect of vitamin D supplementation in the prevention of ARIs from inception to June 2021. Common keywords used for searching were as follows: "vitamin D," for an intervention variable, "respiratory tract infections," for a disease variable, and "randomized controlled trial" for a study design.

\subsection{Data Selection and Quality}

We selected RCTs that met all the following criteria: reported the efficacy of vitamin D supplementation in the prevention of ARIs; reported outcome measures with dichotomous variables. We excluded studies targeting participants in pregnancy and prenatal periods. Regarding studies using shared data from the identical population, we selected a more comprehensive study or a study with a longer follow-up period. Two authors (H.-E. Cho and $\mathrm{H}$. Cho) independently evaluated the suitability of an individual study using the above-described selection criteria. Discrepancies between authors with the selection were solved with discussion and consultation with the third author (S.-K. Myung).

\subsection{Assessment of Risk of Bias}

The risk of bias was estimated based on both the Jadad score [47] and the Cochrane risk of bias tool [48] by two authors (H.-E. Cho and H. Cho). Studies were considered as having high quality if they had $\geq 5$ items in the Jadad scale or $\geq 6$ items in the Cochrane risk of bias tool because the mean score for the Jadad scale was 4.5 and the Cochrane risk of bias tool was 5 . 


\subsection{Main and Subgroup Meta-Analysis}

In the main analysis, we investigated the association between vitamin D supplementation and the incidence of ARIs as a risk. Subgroup analyses were conducted according to various factors as follows: duration of vitamin D supplementation $(\leq 11$ weeks and $>11$ weeks), dosage (daily, weekly, monthly, $>2000 \mathrm{IU}$, and $\leq 2000 \mathrm{IU}$ ), type of disease (URIs and LRIs), number of the study participants ( $>1000$ vs. $\leq 1000)$, region of the study (America, Europe, Asia, and Oceania), mean age ( $\leq 18 \mathrm{vs.}>18)$, supply source for supplements (pharmaceutical company vs. non-pharmaceutical company), use of placebo, and quality of the study (Jadad score and Cochrane risk of bias).

\subsection{Statistical Analysis}

Values in cells of a $2 \times 2$ table based on an intention-to-treat analysis were used to calculate a relative risk (RR) with its $95 \%$ confidence interval (CI) in an individual study. Then, we calculated a pooled RR with its $95 \% \mathrm{CI}$ in the random-effects meta-analysis.

To test the heterogeneity across studies, Higgins $\mathrm{I}^{2}$, which measures the percentage of total variation across studies [49], was used. $\mathrm{I}^{2}$ calculated by a formula as follows

$$
\mathrm{I}^{2}=100 \% \times(\mathrm{Q}-\mathrm{df}) / \mathrm{Q}
$$

where $\mathrm{Q}$ is the Cochrane's heterogeneity statistic and $\mathrm{df}$ means the degree of freedom.

The negative predictive values of the $\mathrm{I}^{2}$ were set at zero. An $\mathrm{I}^{2}$ value ranges from $0 \%$ (no observed heterogeneity) to $100 \%$ (maximal heterogeneity), and those greater than $50 \%$ indicate substantial heterogeneity [50]. In this study, because individual trials were conducted in the different populations, we used a random-effects model meta-analysis.

The publication bias was evaluated by using the Begg's funnel plot and Egger's test. If Begg's funnel plot shows asymmetry or the $p$-value of the Egger's test is below 0.05, it indicates the existence of publication bias in the study. We used Stata MP version 17.0 software package (StataCorp., College Station, TX, USA) for all the statistical analyses.

\section{Results}

\subsection{Identification of Relevant Studies}

Figure 1 shows how we selected relevant articles, out of a total of 390 articles initially searched from the three databases. After excluding 141 duplicated articles, two authors independently reviewed 249 articles based on the title and abstract. Among them, 196 articles that did not meet the pre-determined selection criteria were excluded. For the remaining 53 articles, we reviewed the full text of the trials and excluded 23 articles because of the following reasons: four articles were irrelevant, five were replies or comments, and 14 had insufficient data. A total of 28 randomized double-blind placebo-controlled trials (RDBPCTs) and two open-label, randomized controlled trials (OLRCTs) [12-41] were included in the final analysis.

\subsection{General Characteristics of Trials}

Table 1 shows the general characteristics of the clinical trials included in the final analysis. Studies were published between 2009 and 2021, spanning 12 years. The total number of the study participants were 30,263 with 4259 in an intervention group and 4069 in a control group. The number of the study participants ranged from 49 to 8117 . For studies reporting the information of age, the mean age of the participants was 36.6 years old (from 3 to 81$)$. The main outcome measures were URIs $(n=23)$, LRIs $(n=6)$, and both URIs and LRIs $(n=1)$. The periods of supplementation or follow-up ranged from 1 week to 60 weeks.

The dosage regimens for vitamin D supplements used in the trials were as follows: 300, 400, 500, 600, 1000, 1200, 2000, 4000, 10,000 IU daily, 14,000, 50,000 IU weekly, 60,000, $100,000,120,000,200,000$ IU monthly, 100,000, or 300,000 IU quarterly. 
Out of 28 trials reporting their funding sources, eight trials were supplied vitamin D supplements from pharmaceutical companies. The remaining 20 trials were funded by mainly public or governmental organizations or independent scientific foundations.

\subsection{Association between Vitamin D Supplementation and Prevention of ARIs}

As shown in Figure 2, a random-effects meta-analyses of RCTs showed that vitamin D supplementation did not significantly lower the risk of ARIs (RR 0.96, 95\% CI 0.91-1.01, $\mathrm{I}^{2}=59.0 \%, n=30$ ).

Identified studies from the databases using keywords and bibliographies of relevant articles $(n=390)$ :

PubMed $(n=103)$, EMBASE $(n=41)$, and Cochrane Library $(n=246)$

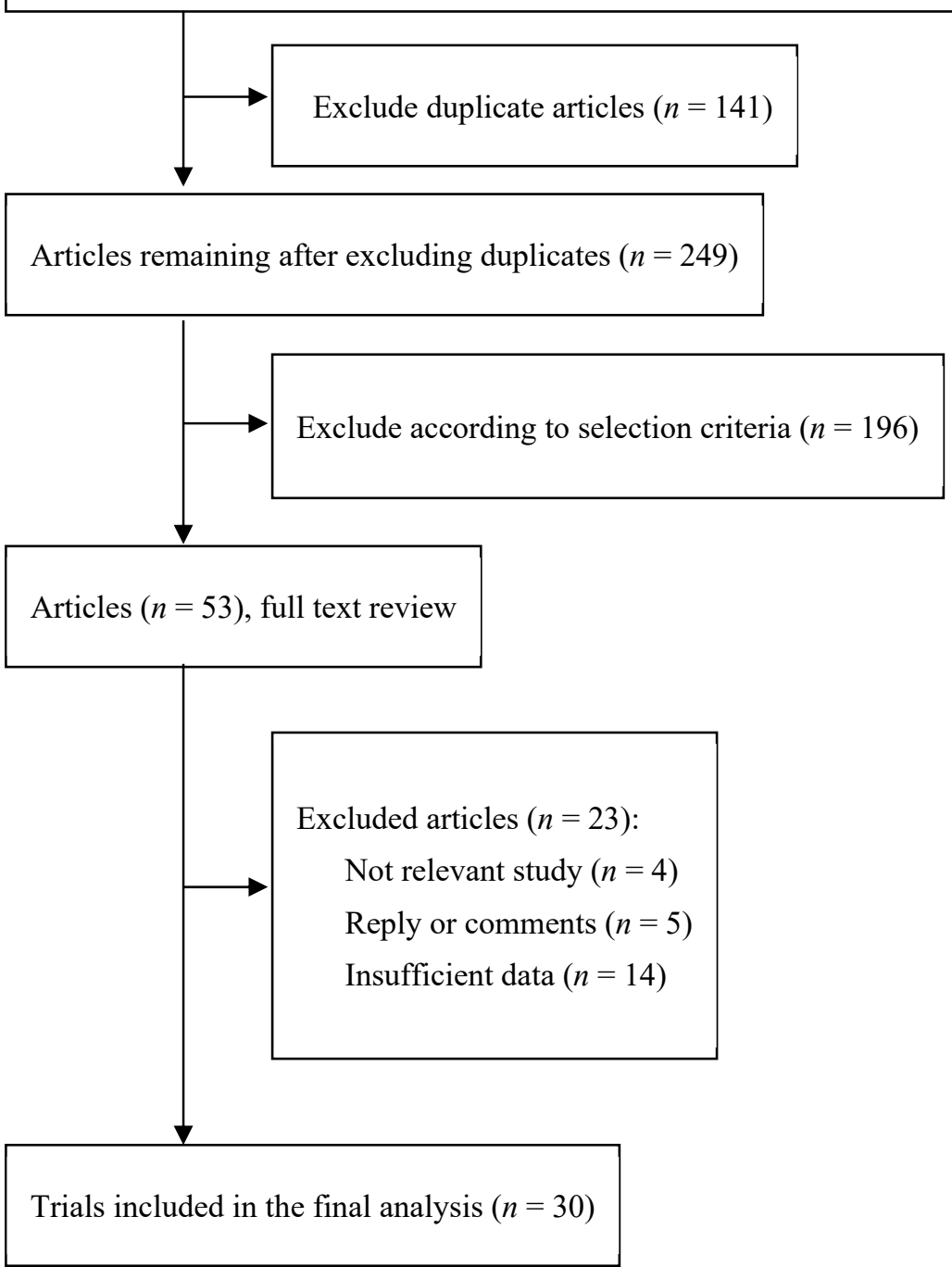

Figure 1. Flow Diagram for selection of relevant clinical trials. 
Table 1. Characteristics of trials included in the final meta-analysis $(n=30)$.

\begin{tabular}{|c|c|c|c|c|c|c|c|c|c|}
\hline & \multirow[t]{2}{*}{ Study } & \multirow[t]{2}{*}{ Region } & \multirow{2}{*}{$\begin{array}{c}\text { Study } \\
\text { Design } \\
\text { (Type of } \\
\text { Prevention) }\end{array}$} & \multirow{2}{*}{$\begin{array}{l}\text { Participants } \\
\text { (Average Age, y; } \\
\text { Women, \%) }\end{array}$} & \multirow{2}{*}{$\begin{array}{c}\text { Duration of } \\
\text { Supplemen- } \\
\text { tation, w } \\
\text { (Follow-Up } \\
\text { Period, w) }\end{array}$} & \multirow{2}{*}{$\begin{array}{l}\text { Intervention } \\
\text { vs. Control }\end{array}$} & \multirow{2}{*}{$\begin{array}{l}\text { Main } \\
\text { Outcome } \\
\text { Measures }\end{array}$} & \multicolumn{2}{|c|}{$\begin{array}{l}\text { No. of Patients with } \\
\text { Acute Respiratory } \\
\text { Infection /No. of } \\
\text { Study Participants }\end{array}$} \\
\hline & & & & & & & & $\begin{array}{l}\text { Supplement } \\
\text { Group }\end{array}$ & $\begin{array}{l}\text { Control } \\
\text { Group }\end{array}$ \\
\hline 1 & $\begin{array}{c}2009, \\
\text { Li-Ng et al. [24] }\end{array}$ & U.S. & RDBPCT & $\begin{array}{c}148 \text { healthy } \\
\text { adults } \\
(59 ; 80)\end{array}$ & $3(3)$ & $\begin{array}{l}\text { Vitamin D } \\
(2000 \mathrm{IU} / \mathrm{d}) \\
\text { vs. placebo }\end{array}$ & $\begin{array}{l}\text { URI } \\
\text { symptoms }\end{array}$ & $28 / 78$ & $29 / 70$ \\
\hline 2 & $\begin{array}{c}2010 \\
\text { Laaksi et al. [13] }\end{array}$ & Finland & RDBPCT & $\begin{array}{l}164 \text { healthy } \\
\text { young men with } \\
\text { military training } \\
\text { (n.a.; 0) }\end{array}$ & $6(6)$ & $\begin{array}{l}\text { Vitamin D } \\
(400 \mathrm{IU} / \mathrm{d}) \\
\text { vs. placebo }\end{array}$ & $\begin{array}{l}\text { common } \\
\text { cold } \\
\text { symptoms }\end{array}$ & $45 / 80$ & $44 / 84$ \\
\hline 3 & $\begin{array}{c}2010, \\
\text { Urashima et al. [14] }\end{array}$ & Japan & RDBPCT & $\begin{array}{l}334 \text { Children } \\
(10 ; 44)\end{array}$ & $4(4)$ & $\begin{array}{l}\text { Vitamin D } \\
(1200 \mathrm{IU} / \mathrm{d}) \\
\text { vs. placebo }\end{array}$ & $\begin{array}{c}\text { Influenza A } \\
\text { infection }\end{array}$ & $18 / 167$ & $31 / 167$ \\
\hline 4 & $\begin{array}{c}2012, \\
\text { Camargo et al. [15] }\end{array}$ & Mongolia & RDBPCT & $\begin{array}{l}244 \text { Children } \\
\quad(10 ; 48)\end{array}$ & $3(3)$ & $\begin{array}{l}\text { Vitamin D } \\
(300 \mathrm{IU} / \mathrm{d})+\text { milk } \\
\text { vs. milk }\end{array}$ & $\begin{array}{l}\text { Acute } \\
\text { respiratory } \\
\text { infection }\end{array}$ & $31 / 141$ & $52 / 103$ \\
\hline 5 & $\begin{array}{l}2012, \\
\text { Manaseki et al. [25] }\end{array}$ & Afghanistan & RDBPCT & $\begin{array}{l}3046 \text { healthy } \\
\text { infants } \\
\text { (n.a.; 48) }\end{array}$ & $18(18)$ & $\begin{array}{c}\text { Vitamin D } \\
(100,000 \mathrm{IU} / 3 \mathrm{~m}) \\
\text { vs. placebo }\end{array}$ & $\begin{array}{l}\text { pneumonia } \\
\text { with CXR }\end{array}$ & $260 / 1524$ & $245 / 1522$ \\
\hline 6 & $\begin{array}{c}2012, \\
\text { Murdoch et al. [26] }\end{array}$ & $\begin{array}{c}\text { New } \\
\text { Zealand }\end{array}$ & RDBPCT & $\begin{array}{l}322 \text { healthy } \\
\text { adults } \\
(48 ; 75)\end{array}$ & $18(18)$ & $\begin{array}{l}\text { Vitamin D } \\
(100,000 \mathrm{IU} / \mathrm{m}) \\
\text { vs. placebo }\end{array}$ & $\begin{array}{c}\text { URI } \\
\text { symptoms }\end{array}$ & $154 / 161$ & $155 / 161$ \\
\hline 7 & $\begin{array}{l}\text { 2013, } \\
\text { Rees et al. [27] }\end{array}$ & n.a. & RDBPCT & $\begin{array}{l}759 \text { healthy } \\
\text { adults with } \\
\text { history of } \\
\text { colorectal } \\
\text { adenoma } \\
(58 ; 42)\end{array}$ & n.a. & $\begin{array}{l}\text { Vitamin D } \\
(1000 \mathrm{IU} / \mathrm{d}) \\
\text { vs. placebo }\end{array}$ & $\begin{array}{c}\text { URI } \\
\text { symptoms }\end{array}$ & $303 / 399$ & $276 / 360$ \\
\hline 8 & $\begin{array}{l}2014, \\
\text { Goodall et al. [16] }\end{array}$ & U.S. & RDBPCT & $\begin{array}{l}492 \text { healthy } \\
\text { students } \\
(19 ; 64)\end{array}$ & $1(1)$ & $\begin{array}{l}\text { Vitamin D } \\
(10,000 \mathrm{IU} / \mathrm{d}) \\
\text { vs. placebo }\end{array}$ & $\begin{array}{c}\text { URI } \\
\text { symptoms }\end{array}$ & $70 / 258$ & $80 / 234$ \\
\hline 9 & $\begin{array}{c}2014, \\
\text { Slow et al. [17] }\end{array}$ & $\begin{array}{l}\text { New } \\
\text { Zealand }\end{array}$ & RDBPCT & $\begin{array}{c}207 \text { non-S. aureus } \\
\text { nasal carriage } \\
\text { adults } \\
(48 ; 75)\end{array}$ & $18(18)$ & $\begin{array}{l}\text { Vitamin D } \\
(100,000 \mathrm{IU} / \mathrm{m}) \\
\text { vs. placebo }\end{array}$ & $\begin{array}{l}\text { S. aureus } \\
\text { nasal } \\
\text { carriage, } \\
\text { culture } \\
\text { positive }\end{array}$ & $28 / 110$ & $17 / 97$ \\
\hline 10 & $\begin{array}{l}2014, \\
\text { Urashima et al. [28] }\end{array}$ & Japan & RDBPCT & $\begin{array}{l}247 \text { adolescents } \\
\text { never have } \\
\text { Influenza A } \\
\text { (n.a.; 34) }\end{array}$ & $2(2)$ & $\begin{array}{l}\text { Vitamin D } \\
(2000 \mathrm{IU} / \mathrm{d}) \\
\text { vs. placebo }\end{array}$ & $\begin{array}{l}\text { Influenza- } \\
\text { like } \\
\text { illness }\end{array}$ & $32 / 148$ & $17 / 99$ \\
\hline 11 & $\begin{array}{c}2015, \\
\text { Bergman et al. [18] }\end{array}$ & Sweden & RDBPCT & $\begin{array}{l}124 \text { patients with } \\
\text { primary immun- } \\
\text { odeficiency } \\
\text { (n.a.; n.a.) }\end{array}$ & $12(12)$ & $\begin{array}{l}\text { Vitamin D } \\
(4000 \mathrm{IU} / \mathrm{d}) \\
\text { vs. placebo }\end{array}$ & $\begin{array}{c}\text { URI } \\
\text { symptoms }\end{array}$ & $26 / 62$ & $39 / 62$ \\
\hline 12 & $\begin{array}{l}2015, \\
\text { Martineau et al. } \\
\text { A (ViDiFlu) [29] }\end{array}$ & U.K. & RDBPCT & $\begin{array}{l}217 \text { residents of } \\
\text { sheltered } \\
\text { accommodation } \\
\text { housing blocks } \\
(67 ; 66)\end{array}$ & $12(12)$ & $\begin{array}{c}\text { Vitamin D } \\
(120,000 \mathrm{IU} / 2 \mathrm{~m}+ \\
400 \mathrm{IU} / \mathrm{d}) \\
\text { vs. } \\
\text { Vitamin D } \\
(400 \mathrm{IU} / \mathrm{d})+ \\
\text { placebo }\end{array}$ & $\begin{array}{c}\text { ARI } \\
\text { symptoms }\end{array}$ & $83 / 125$ & $58 / 92$ \\
\hline 13 & $\begin{array}{l}2015, \\
\text { Martineau et al. } \\
\text { B (ViDiCO) [30] }\end{array}$ & U.K. & RDBPCT & $\begin{array}{c}205 \text { patients with } \\
\text { COPD, } \\
\text { emphysema, } \\
\text { chronic } \\
\text { bronchitis } \\
(65 ; 40)\end{array}$ & $12(12)$ & $\begin{array}{c}\text { Vitamin D } \\
(120,000 \mathrm{IU} / 2 \mathrm{~m}) \\
\text { vs. placebo }\end{array}$ & $\begin{array}{c}\text { URI } \\
\text { symptoms }\end{array}$ & $76 / 102$ & $75 / 103$ \\
\hline 14 & $\begin{array}{l}\text { 2015, } \\
\text { Martineau et al. } \\
\text { C (ViDiAs) [31] }\end{array}$ & U.K. & RDBPCT & $\begin{array}{c}232 \text { patients with } \\
\text { asthma } \\
(48 ; 57)\end{array}$ & $12(12)$ & $\begin{array}{c}\text { Vitamin D } \\
(120,000 \mathrm{IU} / 2 \mathrm{~m}) \\
\text { vs. placebo }\end{array}$ & $\begin{array}{l}\text { URI } \\
\text { symptoms }\end{array}$ & $85 / 115$ & $93 / 117$ \\
\hline 15 & $\begin{array}{l}\text { 2015, } \\
\text { Mayan et al. [32] }\end{array}$ & Israel & RDBPCT & $\begin{array}{l}55 \text { adolescent } \\
\text { swimmers } \\
(15 ; 36)\end{array}$ & $12(12)$ & $\begin{array}{l}\text { Vitamin D } \\
\text { (2000 IU/d) } \\
\text { vs. placebo }\end{array}$ & $\begin{array}{c}\text { URI } \\
\text { symptoms }\end{array}$ & $11 / 28$ & $11 / 27$ \\
\hline
\end{tabular}


Table 1. Cont.

\begin{tabular}{|c|c|c|c|c|c|c|c|c|c|}
\hline & \multirow[t]{2}{*}{ Study } & \multirow[t]{2}{*}{ Region } & \multirow{2}{*}{$\begin{array}{c}\text { Study } \\
\text { Design } \\
\text { (Type of } \\
\text { Prevention) }\end{array}$} & \multirow{2}{*}{$\begin{array}{l}\text { Participants } \\
\text { (Average Age, y; } \\
\text { Women, \%) }\end{array}$} & \multirow{2}{*}{$\begin{array}{c}\text { Duration of } \\
\text { Supplemen- } \\
\text { tation, w } \\
\text { (Follow-Up } \\
\text { Period, w) }\end{array}$} & \multirow{2}{*}{$\begin{array}{l}\text { Intervention } \\
\text { vs. Control }\end{array}$} & \multirow[t]{2}{*}{$\begin{array}{l}\text { Main } \\
\text { Outcome } \\
\text { Measures }\end{array}$} & \multicolumn{2}{|c|}{$\begin{array}{c}\text { No. of Patients with } \\
\text { Acute Respiratory } \\
\text { Infection /No. of } \\
\text { Study Participants }\end{array}$} \\
\hline & & & & & & & & $\begin{array}{l}\text { Supplemen } \\
\text { Group }\end{array}$ & $\begin{array}{l}\text { Control } \\
\text { Group }\end{array}$ \\
\hline 16 & $\begin{array}{c}2016, \\
\text { Denlinger et al. [33] }\end{array}$ & n.a. & RDBPCT & $\begin{array}{l}408 \text { patients with } \\
\text { asthma } \\
\text { (n.a.) }\end{array}$ & $28(28)$ & $\begin{array}{l}\text { Vitamin D } \\
(4000 \mathrm{IU} / \mathrm{d}) \\
\text { vs. placebo }\end{array}$ & $\begin{array}{l}\text { URI } \\
\text { symptoms }\end{array}$ & $161 / 201$ & $139 / 207$ \\
\hline 17 & $\begin{array}{c}\text { 2016, } \\
\text { Gupta et al. [34] }\end{array}$ & India & RDBPCT & $\begin{array}{l}314 \text { children } \\
\text { with pneumonia } \\
\quad(12 \mathrm{~m} ; 30)\end{array}$ & once (6) & $\begin{array}{l}\text { Vitamin D } \\
(100,000 \text { IU) } \\
\text { vs. placebo }\end{array}$ & pneumonia & $39 / 156$ & $36 / 158$ \\
\hline 18 & $\begin{array}{c}\text { 2017, } \\
\text { Aglipay et al. [35] }\end{array}$ & Canada & RDBPCT & $\begin{array}{l}703 \text { healthy } \\
\text { children } \\
(3 ; 42)\end{array}$ & $4-8(4-8)$ & $\begin{array}{c}\text { Vitamin D } \\
(2000 \mathrm{IU} / \mathrm{d}+ \\
400 \mathrm{IU} / \mathrm{d}) \\
\text { vs. Vitamin D } \\
(400 \mathrm{IU} / \mathrm{d})\end{array}$ & URI & $184 / 349$ & $193 / 354$ \\
\hline 19 & $\begin{array}{l}\text { 2017, } \\
\text { Ginde et al. [19] }\end{array}$ & U.S. & RDBPCT & $\begin{array}{l}107 \text { long term } \\
\text { care residents } \\
\quad(81 ; 58)\end{array}$ & $12(12)$ & $\begin{array}{c}\text { Vitamin D } \\
(100,000 \mathrm{IU} / \mathrm{m}) \\
\text { vs. } \\
\text { Vitamin D } \\
(1200 \mathrm{IU} / \mathrm{m})\end{array}$ & $\begin{array}{c}\text { URI } \\
\text { symptoms }\end{array}$ & $17 / 55$ & $24 / 52$ \\
\hline 20 & $\begin{array}{l}\text { 2018, } \\
\text { Brett et al. [36] }\end{array}$ & Canada & OLRCT & $\begin{array}{l}49 \text { healthy } \\
\text { children } \\
(6 ; 47)\end{array}$ & $3(3)$ & $\begin{array}{l}\text { Vitamin D } \\
\text { fortified food } \\
(600 \mathrm{IU} / \mathrm{d}) \\
\text { vs. placebo }\end{array}$ & $\begin{array}{l}\text { common } \\
\text { cold } \\
\text { symptoms }\end{array}$ & $13 / 25$ & $14 / 24$ \\
\hline 21 & $\begin{array}{c}2018, \\
\text { Hibbs et al. [20] }\end{array}$ & U.S. & RDBPCT & $\begin{array}{c}306 \text { preterm black } \\
\text { infants } \\
\text { (n.a.; } 67)\end{array}$ & $6(12)$ & $\begin{array}{l}\text { Vitamin D } \\
(400 \mathrm{IU} / \mathrm{d}) \\
\text { vs. placebo }\end{array}$ & URI & $84 / 153$ & $83 / 153$ \\
\hline 22 & $\begin{array}{c}2018, \\
\text { Shimizu et al. [21] }\end{array}$ & Japan & RDBPCT & $\begin{array}{l}215 \text { healthy } \\
\text { adults } \\
(54 ; 69)\end{array}$ & $16(16)$ & $\begin{array}{l}\text { Vitamin D } \\
(400 \mathrm{IU} / \mathrm{d}) \\
\text { vs. placebo }\end{array}$ & $\begin{array}{l}\text { URI } \\
\text { symptoms }\end{array}$ & $41 / 110$ & $43 / 105$ \\
\hline 23 & $\begin{array}{l}\text { 2018, } \\
\text { Zhou et al. [22] }\end{array}$ & China & OLRCT & $\begin{array}{l}332 \text { healthy } \\
\text { infants } \\
(8 ; 48)\end{array}$ & $4(4)$ & $\begin{array}{c}\text { Vitamin D } \\
(1200 \mathrm{IU} / \mathrm{d}+ \\
400 \mathrm{IU} / \mathrm{d}) \\
\text { vs. } \\
\text { Vitamin D } \\
(400 \mathrm{IU} / \mathrm{d})\end{array}$ & Influenza A & $43 / 164$ & $78 / 168$ \\
\hline 24 & $\begin{array}{l}2019, \\
\text { Arihiro et al. [23] }\end{array}$ & Japan & RDBPCT & $\begin{array}{l}223 \text { patients } \\
\text { with IBD } \\
(45 ; 39)\end{array}$ & $6(6)$ & $\begin{array}{l}\text { Vitamin D } \\
(500 \mathrm{IU} / \mathrm{d}) \\
\text { vs. placebo }\end{array}$ & $\begin{array}{l}\text { URI } \\
\text { symptoms }\end{array}$ & $19 / 108$ & $30 / 115$ \\
\hline 25 & $\begin{array}{l}2019, \\
\text { Loeb et al. [37] }\end{array}$ & Vietnam & RDBPCT & $\begin{array}{l}1300 \text { healthy } \\
\text { children and } \\
\text { adolescent } \\
(9 ; 52)\end{array}$ & $8(8)$ & $\begin{array}{l}\text { Vitamin D } \\
(14,000 \mathrm{IU} / \mathrm{w}) \\
\text { vs. placebo }\end{array}$ & $\begin{array}{c}\text { Influenza A } \\
\text { or B }\end{array}$ & $50 / 650$ & $43 / 650$ \\
\hline 26 & $\begin{array}{l}\text { 2019, } \\
\text { Singh et al. [38] }\end{array}$ & n.a. & OLRCT & $\begin{array}{l}100 \text { children } \\
\text { with pneumonia } \\
\text { (n.a.; 42) }\end{array}$ & $8(12)$ & $\begin{array}{c}\text { Vitamin D } \\
(300,000 / 3 \mathrm{~m})+ \\
\text { milk } \\
\text { vs. placebo + } \\
\text { milk }\end{array}$ & $\begin{array}{l}\text { LRI } \\
\text { symptoms }\end{array}$ & $28 / 50$ & $34 / 50$ \\
\hline 27 & $\begin{array}{c}2020, \\
\text { Camargo et al. [39] }\end{array}$ & $\begin{array}{c}\text { New } \\
\text { Zealand }\end{array}$ & RDBPCT & $\begin{array}{l}5056 \text { healthy } \\
\text { adults } \\
(66 ; 42)\end{array}$ & $19.2(19.2)$ & $\begin{array}{c}\text { Vitamin D } \\
(100,000 \mathrm{IU} / \mathrm{m}) \\
\text { vs. placebo }\end{array}$ & $\begin{array}{c}\text { ARI } \\
\text { symptoms }\end{array}$ & $1882 / 2539$ & $1855 / 2517$ \\
\hline 28 & $\begin{array}{c}2020, \\
\text { Ganmaa et al. [40] }\end{array}$ & Mongolia & RDBPCT & $\begin{array}{l}8117 \text { children } \\
\text { without TB } \\
(9 ; 49)\end{array}$ & $36(36)$ & $\begin{array}{l}\text { Vitamin D } \\
(14,000 \mathrm{IU} / \mathrm{w}) \\
\text { vs. placebo }\end{array}$ & $\begin{array}{c}\text { Pulmonary } \\
\text { TB, QFT } \\
\text { results }\end{array}$ & $147 / 4074$ & $134 / 4043$ \\
\hline 29 & $\begin{array}{c}2020, \\
\text { Sudfeld et al. [41] }\end{array}$ & Tanzania & RDBPCT & $\begin{array}{c}3639 \text { patients } \\
\text { with HIV } \\
\text { with ART } \\
(39 ; 32)\end{array}$ & $12(12)$ & $\begin{array}{c}\text { Vitamin D } \\
(50,000 \mathrm{IU} / \mathrm{w} \\
\text { than } 2000 \mathrm{IU} / \mathrm{d}) \\
\text { vs. placebo }\end{array}$ & $\begin{array}{l}\text { Pulmonary } \\
\text { TB }\end{array}$ & $50 / 1812$ & $64 / 1827$ \\
\hline 30 & $\begin{array}{c}\text { 2021, } \\
\text { Pham et al. [42] }\end{array}$ & Australia & RDBPCT & $\begin{array}{c}2598 \text { healthy } \\
\text { adults } \\
\text { (n.a.; } 51)\end{array}$ & $60(60)$ & $\begin{array}{c}\text { Vitamin D } \\
(60,000 \mathrm{IU} / \mathrm{m}) \\
\text { vs. placebo }\end{array}$ & $\begin{array}{c}\text { ARI } \\
\text { symptoms }\end{array}$ & $410 / 1318$ & $404 / 1280$ \\
\hline
\end{tabular}

n.a., not available; RDBPCT, randomized, double-blind, placebo-controlled trial; OLRCT, open-label, randomized, controlled trial; URI, upper respiratory tract infection; LRI, lower respiratory tract infection; ARI; acute respiratory infection; TB, tuberculosis; CXR, chest X-ray; QFT, QuantiFERON-TB; ART, antiretroviral therapy; HIV, human immunodeficiency virus; A (ViDiFlu), trial of vitamin D supplementation for prevention of Influenza; B (ViDiCO), vitamin D3 supplementation in patients with chronic obstructive pulmonary disease; C (ViDiAs), vitamin D3 supplementation in adults with asthma. 


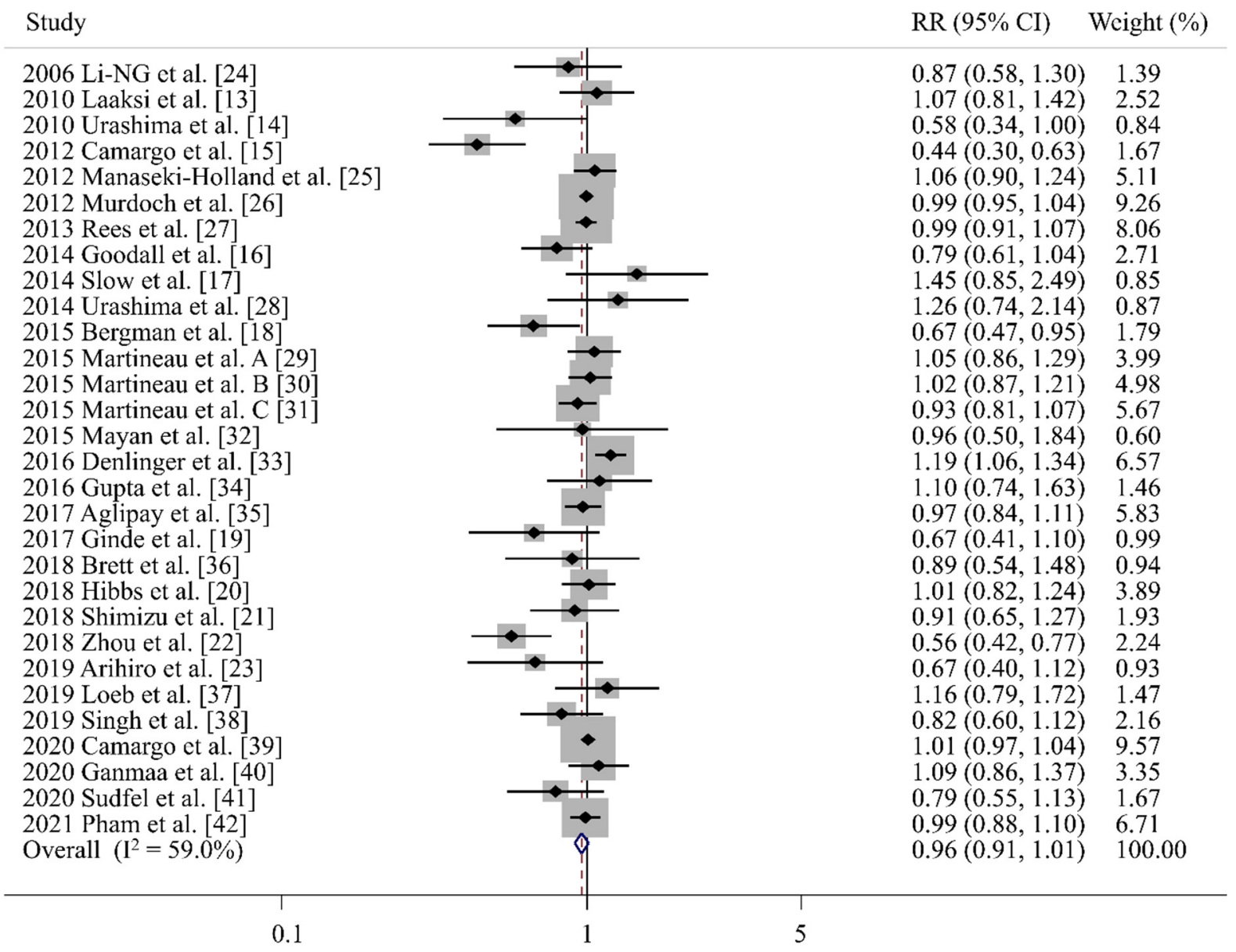

Figure 2. Efficacy of vitamin D supplements in prevention of acute respiratory infections in a meta-analysis of randomized controlled trials $(n=30)$ [13-42]. RR, relative risk; CI, confidence interval; A, trial of vitamin D supplementation for prevention of Influenza (ViDiFlu); B, vitamin D3 supplementation in patients with chronic obstructive pulmonary disease (ViDiCO); C, vitamin D3 supplementation in adults with asthma (ViDiAs).

\subsection{Quality Assessment}

The mean score of all the trials was 4.5 (Table 2) and 5 (Table 3) in the quality assessment based on the Jadad scale and the Cochrane risk of bias tool, respectively. Nineteen studies [13-16,19-21,25-28,30,34,35,37,39,41,42] were considered as having high quality in the Jadad scale, while $11[17,18,22-24,29,32,33,36,38,40]$ were considered as having low quality (Table 2). Fourteen studies [14,19,23,25,26,28,29,31,34,35,37,39-41] were high-quality studies in the Cochrane risk of bias tool, while the remaining 16 [13,15-18,20-24,27,30,32,33,36,38,42] were low-quality studies (Table 3).

\subsection{Subgroup Meta-Analysis and the Publication Bias}

Table 4 shows that vitamin D supplementation was efficacious in the prevention of ARIs in the subgroup meta-analyses by several factors as follows: duration of the study $\leq 11$ weeks, daily supplementation, low vitamin $\mathrm{D}$ dosage $\leq 2000 \mathrm{IU}$, and the number of the study population $\leq 1000$. 
Table 2. Methodological quality of trials based on the Jadad scale $(n=30)$.

\begin{tabular}{|c|c|c|c|c|c|c|c|}
\hline & Study & Randomization & $\begin{array}{c}\text { Description of } \\
\text { Randomization } \\
\text { Methods }\end{array}$ & $\begin{array}{l}\text { DOUBLE- } \\
\text { BLIND }\end{array}$ & $\begin{array}{c}\text { Using } \\
\text { Identical } \\
\text { Placebo }\end{array}$ & $\begin{array}{l}\text { Follow-Up } \\
\text { Reporting }\end{array}$ & Total Score \\
\hline 1 & $\begin{array}{c}2009, \\
\text { Li-Ng et al. [24] }\end{array}$ & 1 & 1 & 1 & 0 & 1 & 4 \\
\hline 2 & $\begin{array}{c}\text { 2010, } \\
\text { Laaksi et al. [13] }\end{array}$ & 1 & 1 & 1 & 1 & 1 & 5 \\
\hline 3 & $\begin{array}{c}\text { 2010, } \\
\text { Urashima et al. [14] }\end{array}$ & 1 & 1 & 1 & 1 & 1 & 5 \\
\hline 4 & $\begin{array}{c}2012, \\
\text { Camargo et al. [15] }\end{array}$ & 1 & 1 & 1 & 1 & 1 & 5 \\
\hline 5 & $\begin{array}{c}2012, \\
\text { Manaseki et al. [25] }\end{array}$ & 1 & 1 & 1 & 1 & 1 & 5 \\
\hline 6 & $\begin{array}{c}2012, \\
\text { Murdoch et al. [26] }\end{array}$ & 1 & 1 & 1 & 1 & 1 & 5 \\
\hline 7 & $\begin{array}{c}\text { 2013, } \\
\text { Rees et al. [27] }\end{array}$ & 1 & 1 & 1 & 1 & 1 & 5 \\
\hline 8 & $\begin{array}{c}\text { 2014, } \\
\text { Goodall et al. [16] }\end{array}$ & 1 & 1 & 1 & 1 & 1 & 5 \\
\hline 9 & $\begin{array}{l}\text { 2014, } \\
\text { Slow et al. [17] }\end{array}$ & 1 & 0 & 1 & 1 & 1 & 4 \\
\hline 10 & $\begin{array}{c}2014 \\
\text { Urashima et al. [28] }\end{array}$ & 1 & 1 & 1 & 1 & 1 & 5 \\
\hline 11 & $\begin{array}{c}2015, \\
\text { Bergman et al. [18] }\end{array}$ & 1 & 1 & 1 & 0 & 1 & 4 \\
\hline 12 & $\begin{array}{c}2015, \\
\text { Martineau et al. } \\
\text { A (ViDiFlu) [29] }\end{array}$ & 1 & 0 & 1 & 0 & 1 & 3 \\
\hline 13 & $\begin{array}{l}\text { 2014, } \\
\text { Martineau et al. } \\
\text { B (ViDiCO) }[30]\end{array}$ & 1 & 1 & 1 & 1 & 1 & 5 \\
\hline 14 & $\begin{array}{l}\text { 2015, } \\
\text { Martineau et al. } \\
\text { C (ViDiAs) [31] }\end{array}$ & 1 & 1 & 1 & 1 & 1 & 5 \\
\hline 15 & $\begin{array}{c}\text { 2015, } \\
\text { Mayan et al. [32] }\end{array}$ & 1 & 0 & 1 & 1 & 1 & 4 \\
\hline 16 & $\begin{array}{c}\text { 2016, } \\
\text { Denlinger et al. [33] }\end{array}$ & 1 & 0 & 1 & 0 & 1 & 3 \\
\hline 17 & $\begin{array}{c}\text { 2016, } \\
\text { Gupta et al. [34] }\end{array}$ & 1 & 1 & 1 & 1 & 1 & 5 \\
\hline 18 & $\begin{array}{c}\text { 2017, } \\
\text { Aglipay et al. [35] }\end{array}$ & 1 & 1 & 1 & 1 & 1 & 5 \\
\hline 19 & $\begin{array}{l}\text { 2017, } \\
\text { Ginde et al. [19] }\end{array}$ & 1 & 1 & 1 & 1 & 1 & 5 \\
\hline 20 & $\begin{array}{l}\text { 2018, } \\
\text { Brett et al. [36] }\end{array}$ & 1 & 0 & 0 & 0 & 0 & 1 \\
\hline 21 & $\begin{array}{l}2018, \\
\text { Hibbs et al. [20] }\end{array}$ & 1 & 1 & 1 & 1 & 1 & 5 \\
\hline 22 & $\begin{array}{c}\text { 2018, } \\
\text { Shimizu et al. [21] }\end{array}$ & 1 & 0 & 1 & 1 & 1 & 5 \\
\hline 23 & $\begin{array}{c}\text { 2018, } \\
\text { Zhou et al. [22] }\end{array}$ & 1 & 0 & 0 & 0 & 1 & 2 \\
\hline
\end{tabular}


Table 2. Cont.

\begin{tabular}{|c|c|c|c|c|c|c|c|}
\hline & Study & Randomization & $\begin{array}{l}\text { Description of } \\
\text { Randomization } \\
\text { Methods }\end{array}$ & $\begin{array}{l}\text { DOUBLE- } \\
\text { BLIND }\end{array}$ & $\begin{array}{l}\text { Using } \\
\text { Identical } \\
\text { Placebo }\end{array}$ & $\begin{array}{l}\text { Follow-Up } \\
\text { Reporting }\end{array}$ & Total Score \\
\hline 24 & $\begin{array}{l}\text { 2019, } \\
\text { Arihiro et al. [23] }\end{array}$ & 1 & 1 & 1 & 0 & 1 & 4 \\
\hline 25 & $\begin{array}{c}2019, \\
\text { Loeb et al. [37] }\end{array}$ & 1 & 1 & 1 & 1 & 1 & 5 \\
\hline 26 & $\begin{array}{l}\text { 2019, } \\
\text { Singh et al. [38] }\end{array}$ & 1 & 0 & 0 & 1 & 1 & 3 \\
\hline 27 & $\begin{array}{c}\text { 2020, } \\
\text { Camargo et al. [39] }\end{array}$ & 1 & 1 & 1 & 1 & 1 & 5 \\
\hline 28 & $\begin{array}{c}2020, \\
\text { Ganmaa et al. [40] }\end{array}$ & 1 & 0 & 1 & 0 & 1 & 3 \\
\hline 29 & $\begin{array}{c}2020, \\
\text { Sudfeld et al. [41] }\end{array}$ & 1 & 1 & 1 & 1 & 1 & 5 \\
\hline 30 & $\begin{array}{c}\text { 2021, } \\
\text { Pham et al. [42] }\end{array}$ & 1 & 1 & 1 & 1 & 1 & 5 \\
\hline
\end{tabular}

Table 3. Methodological quality of trials based on the Cochrane risk of bias tool $(n=30)$.

\begin{tabular}{|c|c|c|c|c|c|c|c|c|}
\hline Study & $\begin{array}{c}\text { Random } \\
\text { Sequence } \\
\text { Generation }\end{array}$ & $\begin{array}{l}\text { Allocation } \\
\text { Concealment }\end{array}$ & $\begin{array}{c}\text { Blinding of } \\
\text { Participants } \\
\text { and Personnel }\end{array}$ & $\begin{array}{l}\text { Blinding of } \\
\text { Outcome } \\
\text { Assessment }\end{array}$ & $\begin{array}{l}\text { Incomplete } \\
\text { Outcome Data }\end{array}$ & $\begin{array}{l}\text { Selective } \\
\text { Reporting }\end{array}$ & Other Bias & $\begin{array}{l}\text { No. of Low } \\
\text { Risk of Bias }\end{array}$ \\
\hline $\begin{array}{c}2009, \\
\text { Li-Ng et al. [24] }\end{array}$ & Low & Unclear & Low & High & Low & Low & Low & 5 \\
\hline $\begin{array}{c}\text { 2010, } \\
\text { Laaksi et al. [13] }\end{array}$ & Low & Low & Unclear & Low & Unclear & Low & Low & 5 \\
\hline $\begin{array}{l}2010, \\
\text { Urashima et al. [14] }\end{array}$ & Low & Low & Low & Low & Unclear & Low & Low & 6 \\
\hline $\begin{array}{c}2012, \\
\text { Camargo et al. [15] }\end{array}$ & Low & Low & Low & Unclear & Low & Unclear & Low & 5 \\
\hline $\begin{array}{c}\text { 2012, } \\
\text { Manaseki et al. [25] }\end{array}$ & Low & Low & Low & Low & Unclear & Low & Low & 6 \\
\hline $\begin{array}{c}\text { 2012, } \\
\text { Murdoch et al. [26] }\end{array}$ & Low & Low & Low & Low & Low & Low & Low & 7 \\
\hline $\begin{array}{l}\text { 2013, } \\
\text { Rees et al. [27] }\end{array}$ & Low & Low & Low & High & Unclear & Low & Low & 5 \\
\hline $\begin{array}{l}\text { 2014, } \\
\text { Goodall et al. [16] }\end{array}$ & Low & Low & Low & Low & Unclear & Unclear & Low & 5 \\
\hline $\begin{array}{l}\text { 2014, } \\
\text { Slow et al. [17] }\end{array}$ & Unclear & Unclear & Low & Low & Low & Low & Low & 5 \\
\hline $\begin{array}{l}2014, \\
\text { Urashima et al. [28] }\end{array}$ & Low & Low & Low & Unclear & Low & Low & Low & 6 \\
\hline $\begin{array}{c}2015, \\
\text { Bergman et al. [18] }\end{array}$ & Low & Low & Low & Low & Unclear & Unclear & Low & 5 \\
\hline $\begin{array}{c}2015, \\
\text { Martineau et al. } \\
\text { A (ViDiFlu) [29] }\end{array}$ & Low & Unclear & Low & Low & Low & Low & Low & 6 \\
\hline $\begin{array}{l}2014, \\
\text { Martineau et al. } \\
\text { B (ViDiCO) [30] }\end{array}$ & Low & Unclear & Low & Unclear & Low & Low & Low & 5 \\
\hline $\begin{array}{l}2015, \\
\text { Martineau et al. } \\
\text { C (ViDiAs) [31] }\end{array}$ & Low & Low & Low & Unclear & Low & Low & Low & 6 \\
\hline $\begin{array}{c}2015, \\
\text { Mayan et al. [32] }\end{array}$ & Unclear & High & Unclear & Unclear & Unclear & Low & Low & 2 \\
\hline $\begin{array}{c}\text { 2016, } \\
\text { Denlinger et al. [33] }\end{array}$ & Unclear & Unclear & Unclear & Unclear & Unclear & Low & Low & 2 \\
\hline
\end{tabular}


Table 3. Cont.

\begin{tabular}{|c|c|c|c|c|c|c|c|c|}
\hline Study & $\begin{array}{c}\text { Random } \\
\text { Sequence } \\
\text { Generation }\end{array}$ & $\begin{array}{c}\text { Allocation } \\
\text { Concealment }\end{array}$ & $\begin{array}{c}\text { Blinding of } \\
\text { Participants } \\
\text { and Personnel }\end{array}$ & $\begin{array}{l}\text { Blinding of } \\
\text { Outcome } \\
\text { Assessment }\end{array}$ & $\begin{array}{l}\text { Incomplete } \\
\text { Outcome Data }\end{array}$ & $\begin{array}{l}\text { Selective } \\
\text { Reporting }\end{array}$ & Other Bias & $\begin{array}{l}\text { No. of Low } \\
\text { Risk of Bias }\end{array}$ \\
\hline $\begin{array}{c}\text { 2016, } \\
\text { Gupta et al. [34] }\end{array}$ & Low & Low & Low & Low & Low & Low & Low & 7 \\
\hline $\begin{array}{c}\text { 2017, } \\
\text { Aglipay et al. [35] }\end{array}$ & Low & Low & Low & Low & Unclear & Low & Low & 6 \\
\hline $\begin{array}{l}\text { 2017, } \\
\text { Ginde et al. [19] }\end{array}$ & Low & Low & Low & Low & Low & Low & Low & 7 \\
\hline $\begin{array}{c}\text { 2018, } \\
\text { Brett et al. [36] }\end{array}$ & Unclear & Unclear & Unclear & Unclear & Unclear & Low & Low & 2 \\
\hline $\begin{array}{c}\text { 2018, } \\
\text { Hibbs et al. et al. [20] }\end{array}$ & Low & Unclear & Low & Unclear & Low & Low & Low & 5 \\
\hline $\begin{array}{c}2018, \\
\text { Shimizu et al. [21] }\end{array}$ & Low & Low & Low & Unclear & Unclear & Low & Low & 5 \\
\hline $\begin{array}{l}\text { 2018, } \\
\text { Zhou et al. [22] }\end{array}$ & Unclear & Unclear & Unclear & Unclear & Unclear & Low & Low & 2 \\
\hline $\begin{array}{c}\text { 2019, } \\
\text { Arihiro et al. [23] }\end{array}$ & Low & Low & Low & Low & Low & Low & Low & 7 \\
\hline $\begin{array}{l}\text { 2019, } \\
\text { Loeb et al. [37] }\end{array}$ & Low & Low & Low & Low & Unclear & Low & Low & 6 \\
\hline $\begin{array}{c}\text { 2019, } \\
\text { Singh et al. }\end{array}$ & Unclear & Low & Unclear & Unclear & Unclear & Low & Low & 3 \\
\hline $\begin{array}{c}2020, \\
\text { Camargo et al. [39] }\end{array}$ & Low & Low & Low & Unclear & Low & Low & Low & 6 \\
\hline $\begin{array}{c}\text { 2020, } \\
\text { Ganmaa et al. [40] }\end{array}$ & Low & Unclear & Low & Low & Low & Low & Low & 6 \\
\hline $\begin{array}{c}2020 \\
\text { Sudfeld et al. [41] }\end{array}$ & Low & Low & Low & Low & Low & Low & Low & 7 \\
\hline $\begin{array}{l}\text { 2021, } \\
\text { Pham et al. [42] }\end{array}$ & Low & Low & Low & Unclear & Unclear & Low & Low & 5 \\
\hline
\end{tabular}

Table 4. Vitamin D supplementation in prevention of acute respiratory infections in the subgroup meta-analysis of randomized controlled trials by various factors.

\begin{tabular}{|c|c|c|c|}
\hline Factors & No. of Trials & Summary RR (95\% CI) & Heterogeneity, $\mathrm{I}^{2}(\%)$ \\
\hline All & 30 & $0.96(0.91-1.01)$ & 59.0 \\
\hline \multicolumn{4}{|c|}{ Duration of Vitamin D supplementation } \\
\hline Long term & 15 & $1.01(0.9-1.06)$ & 38.1 \\
\hline Short term & 13 & $0.83(0.71-0.97) *$ & 66.8 \\
\hline \multicolumn{4}{|l|}{ Jadad score } \\
\hline High quality & 9 & $0.88(0.73-1.05)$ & 68.7 \\
\hline Low quality & 4 & $0.71(0.57-0.89) *$ & 26.7 \\
\hline \multicolumn{4}{|l|}{ Cochrane ROB } \\
\hline High quality & 5 & $0.93(0.74-1.16)$ & 43.6 \\
\hline Low quality & 8 & $0.78(0.64-0.97) *$ & 72.9 \\
\hline \multicolumn{4}{|l|}{ Regimen } \\
\hline Daily & 15 & $0.83(0.73-0.95) *$ & 69.1 \\
\hline \multicolumn{4}{|l|}{ Jadad score } \\
\hline High quality & 10 & $0.89(0.78-1.02)$ & 67.0 \\
\hline Low quality & 5 & $0.69(0.58-0.82) *$ & 0.0 \\
\hline
\end{tabular}


Table 4. Cont.

\begin{tabular}{|c|c|c|c|}
\hline Factors & No. of Trials & Summary RR (95\% CI) & Heterogeneity, $\mathrm{I}^{2}(\%)$ \\
\hline \multicolumn{4}{|l|}{ Cochrane ROB } \\
\hline High quality & 4 & $0.87(0.66-1.15)$ & 51.0 \\
\hline Low quality & 11 & $0.81(0.69-0.96)^{*}$ & 74.9 \\
\hline Weekly & 3 & $1.10(0.95-1.26)$ & 25.0 \\
\hline Monthly & 10 & $1.00(0.98-1.02)$ & 0.0 \\
\hline \multicolumn{4}{|l|}{ Dose } \\
\hline High does (>2000 IU) & 8 & $0.95(0.88-1.02)$ & 57.3 \\
\hline Low dose ( $\leq 2000 \mathrm{IU})$ & 20 & $0.92(0.85-1.00) *(0.997)$ & 59.5 \\
\hline \multicolumn{4}{|l|}{ Type of Disease } \\
\hline URI & 24 & $0.97(0.91-1.03)$ & 53.9 \\
\hline LRI & 7 & $1.00(0.91-1.11)$ & 0.0 \\
\hline \multicolumn{4}{|l|}{ Number of study participants } \\
\hline$>1000$ & 6 & $1.00(0.98-1.04)$ & 0.0 \\
\hline$\leq 1000$ & 24 & $0.92(0.85-0.99) *$ & 68.7 \\
\hline \multicolumn{4}{|l|}{ Region } \\
\hline America (Canada, U.S.) & 6 & $0.93(0.84-1.03)$ & 0.0 \\
\hline $\begin{array}{c}\text { Europe } \\
\text { (Finland, Sweden, UK) }\end{array}$ & 5 & $0.97(0.86-1.09)$ & 36.9 \\
\hline $\begin{array}{c}\text { Asia } \\
\text { (Afghanistan, China, } \\
\text { India, Israel, Japan, } \\
\text { Mongolia, Vietnam) }\end{array}$ & 11 & $0.85(0.69-1.05)$ & 74.3 \\
\hline $\begin{array}{c}\text { Oceania } \\
\text { (Australia, New Zealand) }\end{array}$ & 4 & $1.00(0.98-1.03$ & 0.0 \\
\hline \multicolumn{4}{|l|}{ Type of prevention } \\
\hline Primary prevention & 26 & $0.94(0.89-0.99) *$ & 58.5 \\
\hline Secondary prevention & 4 & $1.05(0.92-1.21)$ & 59.2 \\
\hline \multicolumn{4}{|l|}{ Mean age } \\
\hline Children & 12 & $0.87(0.75-1.02)$ & 70.9 \\
\hline Adults & 18 & $0.99(0.95-1.04)$ & 41.1 \\
\hline \multicolumn{4}{|l|}{ Funding source } \\
\hline Pharmaceutical company & 8 & $0.99(0.93-1.04)$ & 0.0 \\
\hline Not pharmaceutical company & 22 & $0.94(0.87-1.00)$ & 69.9 \\
\hline Use of placebo & 29 & $0.98(0.94-1.02)$ & 44.2 \\
\hline \multicolumn{4}{|l|}{ Quality } \\
\hline \multicolumn{4}{|l|}{ Jadad score } \\
\hline High quality $(\geq 5)$ & 18 & $1.00(0.97-1.02)$ & 0.0 \\
\hline Low quality $(<5)$ & 12 & $0.85(0.69-1.04)$ & 80.6 \\
\hline \multicolumn{4}{|l|}{ Cochrane ROB } \\
\hline High quality (>5) & 14 & $1.00(0.96-1.03)$ & 10.9 \\
\hline Low quality $(\leq 5)$ & 16 & $0.90(0.81-1.01)$ & 73.5 \\
\hline
\end{tabular}

ARI, acute respiratory infection; RCT, randomized controlled trials; RR, relative risk; ROB, risk of bias; URI, upper respiratory tract infection; LRI, lower respiratory tract infection; CI, confidence interval. * Indicates a statistically significant association.

Daily supplementation of vitamin D significantly decreased the risk of ARIs (RR 0.83, $95 \%$ CI $0.73-0.95, \mathrm{I}^{2}=69.1 \%, n=15$, Figure 3 ), while its weekly and monthly supplementation showed no significant association. However, in the subgroup meta-analysis of high-quality studies, beneficial effects of daily vitamin D were not observed (RR 0.89, 95\% 
CI, $0.78-1.02, \mathrm{I}^{2}=67.0 \%, n=10$, assessed by the Jadad scale, Figure 3 ; RR $0.86,95 \% \mathrm{CI}$, $0.65-1.15, \mathrm{I}^{2}=51.0 \%, n=4$, assessed by the Cochrane's risk of bias tool, Figure 4 ), while beneficial effects remained in low-quality studies (Figures 3 and 4).

In the subgroup meta-analysis by the duration of vitamin D supplementation, the short-term use of vitamin D supplements showed a significant decreased risk of ARIs in the short-term (RR 0.83, 95\% CI, 0.71-0.97, $\mathrm{I}^{2}=66.8 \%, n=13$, Table 4 ). Similar to daily supplementation of vitamin $\mathrm{D}$, beneficial effects disappeared in the subgroup meta-analysis of high-quality studies (RR $0.88,95 \%$ CI, $0.73-1.05, \mathrm{I}^{2}=68.7 \%, n=9$, assessed by the Jadad scale; RR $0.93,95 \%$ CI, 0.74-1.16, $\mathrm{I}^{2}=43.6 \%, n=5$, assessed by the Cochrane's risk of bias tool, Table 4), while beneficial effects remained in low-quality studies (Table 4).

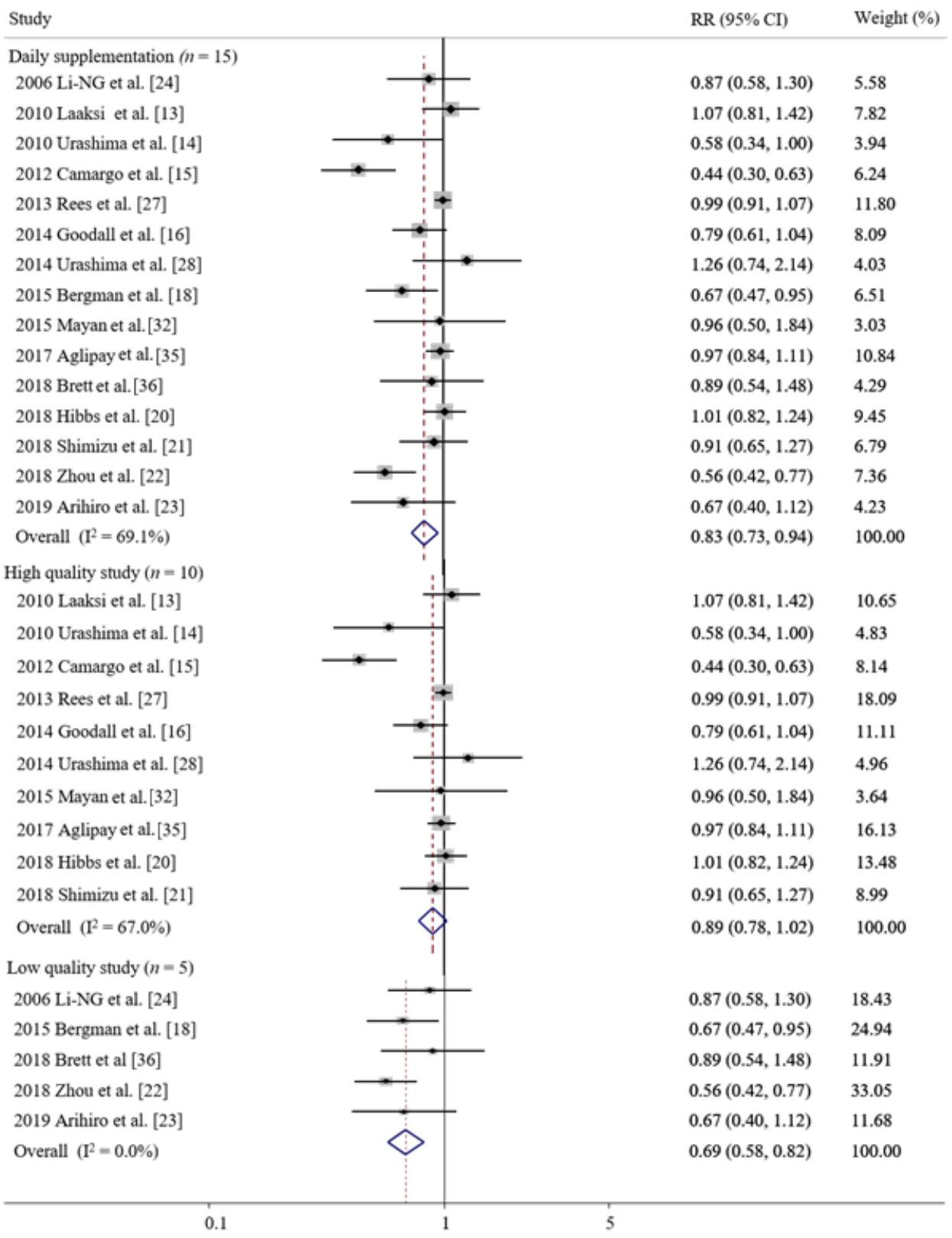

Figure 3. Efficacy of daily supplementation of vitamin D in prevention of acute respiratory infections and its efficacy in subgroup meta-analysis by quality of the study assessed by the Jadad scale [13-16,18,20-24,27,28,32,35,36]. RR, relative risk; CI, confidence interval. 


\begin{tabular}{|c|c|c|}
\hline Study & $\operatorname{RR}(95 \% \mathrm{CI})$ & Weight (\%) \\
\hline \multicolumn{3}{|l|}{ High Quality $(n=4)$} \\
\hline 2010 Urashima et al. [14] & $0.58(0.34,1.00)$ & 17.88 \\
\hline 2014 Urashima et al. [28] & $1.26(0.74,2.14)$ & 18.29 \\
\hline 2017 Aglipay et al.[35] & $0.97(0.84,1.11)$ & 44.67 \\
\hline 2019 Arihiro et al. [23] & $0.67(0.40,1.12)$ & 19.15 \\
\hline Overall $\left(\mathrm{I}^{2}=51.0 \%\right)$ & $0.86(0.65,1.15)$ & 100.00 \\
\hline \multicolumn{3}{|l|}{ Low Quality $(n=11)$} \\
\hline 2006 Li-NG et al. [24] & $0.87(0.58,1.30)$ & 7.67 \\
\hline 2010 Laaksi et al. [13] & $1.07(0.81,1.42)$ & 10.10 \\
\hline 2012 Camargo et al. [15] & $0.44(0.30,0.63)$ & 8.42 \\
\hline 2013 Rees et al. [27] & $0.99(0.91,1.07)$ & 13.76 \\
\hline 2014 Goodall et al. [16] & $0.79(0.61,1.04)$ & 10.38 \\
\hline 2015 Bergman et al. [18] & $0.67(0.47,0.95)$ & 8.71 \\
\hline 2015 Mayan et al. [32] & $0.96(0.50,1.84)$ & 4.50 \\
\hline 2018 Brett et al. [36] & $0.89(0.54,1.48)$ & 6.12 \\
\hline 2018 Hibbs et al. [20] & $1.01(0.82,1.24)$ & 11.69 \\
\hline 2018 Shimizu et al. [21] & $0.91(0.65,1.27)$ & 9.02 \\
\hline 2018 Zhou et al. [22] & $0.56(0.42,0.77)$ & 9.63 \\
\hline Overall $\left(\mathrm{I}^{2}=74.9 \%\right)$ & $0.81(0.69,0.96)$ & 100.00 \\
\hline
\end{tabular}

Figure 4. Efficacy of daily supplementation of vitamin D in prevention of acute respiratory infections in subgroup meta-analysis by quality of the study assessed by Cochrane's risk of bias tool [13-16,18,20-24,27,28,32,35,36]. RR, relative risk; CI, confidence interval.

As shown in Figure 5, publication bias was observed: the Begg's funnel plot was asymmetrical, and the Egger's $p$ for bias was $0.048(p<0.05)$.

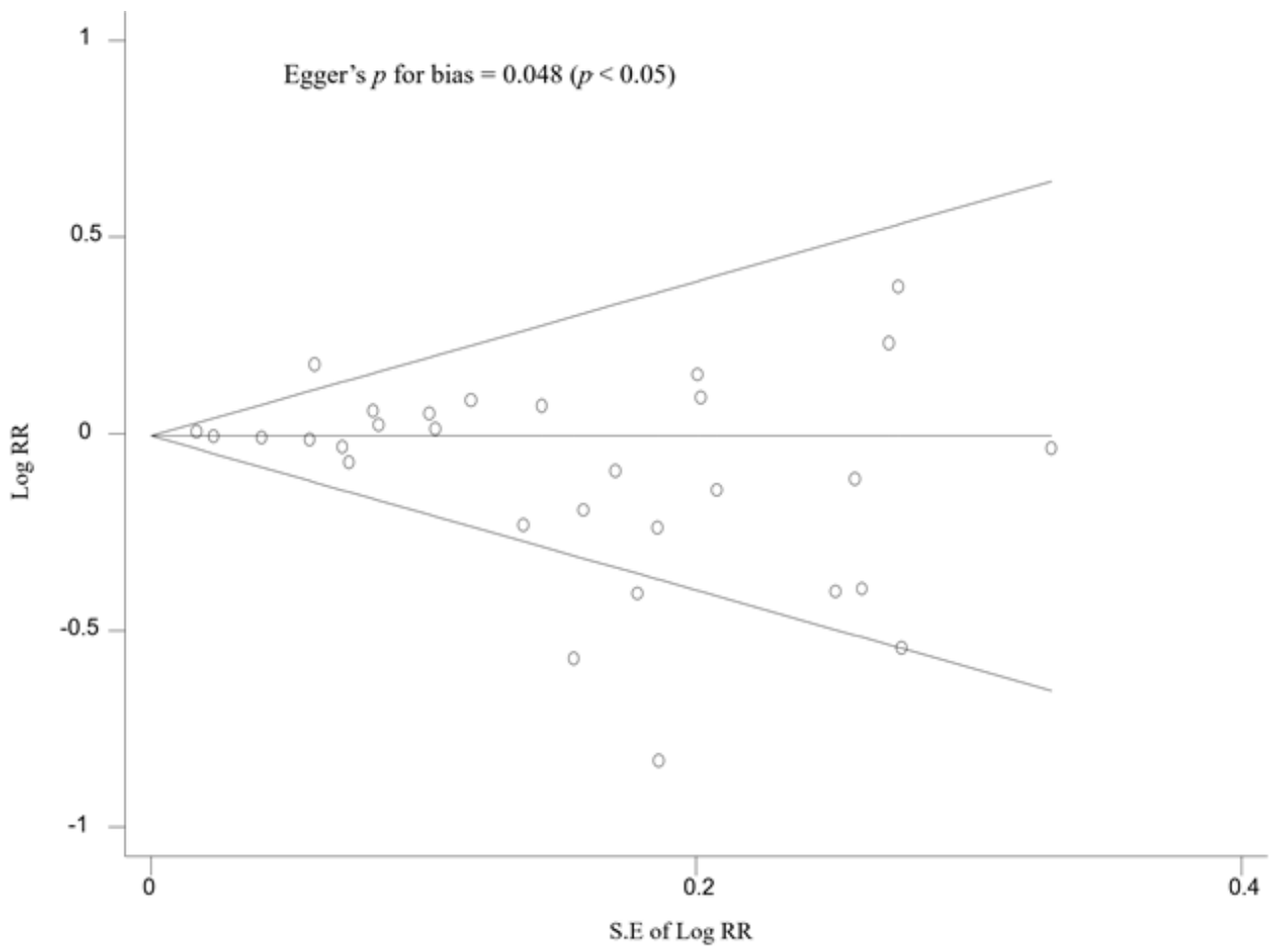

Figure 5. Begg's funnel plot and Egger's test for identifying publication bias of randomized controlled trials. RR, relative risk; S.E, standard error. 


\section{Discussion}

In the current study, we found that the use of vitamin D supplements had no preventive effect on ARIs in the meta-analysis of 30 RCTs. Vitamin D supplementation was efficacious in the prevention of ARIs in the subgroup meta-analyses in daily supplementation and its short-term use. However, the subgroup meta-analyses of the high-quality studies in each category showed that the use of vitamin D supplements has no statistically significant effect in the prevention of ARIs.

There are several biological mechanisms that could explain the preventive effect of vitamin D supplements on ARIs. It has been reported that vitamin D modulates both the adaptive immune and innate immune systems from in vitro studies and animal studies. First, vitamin D could work as a direct and indirect regulator of T cells [7]. Vitamin D regulates $\mathrm{T}$ cells directly by inhibiting $\mathrm{T}$ cell proliferation, Interleukin-2 (IL-2) and Interferon- $\gamma$ (INF- $\gamma$ ) transcription, and IL-17 secretion by helper T 17 cells. Additionally, the vitamin D receptor (VDR) is expressed in both the innate and the adaptive immune cells [7]. The VDR mediates 1,25(OH)2D to suppress helper T 1 cell proliferation that produces inflammatory cytokines, thus decreasing the production of INF- $\gamma$ and IL-2 [51,52]. Moreover, vitamin D induces the development of IL-10 and regulatory T cells [8]. Second, vitamin D fortifies the antibacterial responses of the innate immune response by the toll-like receptors (TLRs) and the 1,25(OH)2D/VDR signaling [7]. The TLRs, which are expressed on macrophages, polymorphonuclear cells, monocytes, and epithelial cells play a key role in the innate immune system [50]. Some of the antimicrobial peptides that demonstrate antiviral effects are associated with the TLRs, and their expression is affected by 1,25(OH)2D [7,50]. In addition, several TLRs are affected by the VDR stimulation [50]. Finally, the gene expression of the antibacterial agents, cathelicidin, and human $\beta$-defensin are induced by $1,25(\mathrm{OH}) 2 \mathrm{D} / \mathrm{VDR}$ signaling [7]. Cathelicidin is an antimicrobial peptide induced by the TLR $1 / 2$ activation, and human $\beta$-defensin acts as a chemoattractant for neutrophils and monocytes [50]. In the animal study, the lungs of the 25(OH)D3-fed mice had a significantly lower viral titer than the lungs of the control mice. After influenza virus infection, the proinflammatory cytokines, IL-5 and INF- $\gamma$, significantly decreased in 25(OH)D3-fed mice compared with the control mice. 25(OH)D3 was found to reduce viral replication and inflammatory cytokines, and then decreased the clinical manifestation of influenza virus infection in a mouse model [11]. In other words, vitamin D deficiency is associated with an increased risk of infections of bacterial and viral origin through decreased innate immunity [53].

In the meantime, previous RCTs and meta-analyses have reported inconsistent findings about the preventive effect of vitamin D supplements on ARIs [6,43-46]. Among them, only one study reported consistent findings with ours [42], and the others reported a preventive effect of vitamin D on ARIs [6,44-46]. Xiao et al.'s [43] systematic review in 2015 concluded that there was no efficacy of vitamin D supplementation for the prevention of childhood ARIs. Martineau et al. [44] and Pham et al. [45] reported that high levels of serum 25(OH)D are associated with the prevention of ARIs. Abioye et al. [6] reported that micronutrients including vitamin D, vitamin C, and zinc reduced the occurrence of ARIs and the duration of the symptoms. Jolliffe et al. [46] suggested that although the heterogeneity across the trial was significant, the vitamin D supplementation slightly reduced the risk of ARIs compared to the control group.

Compared to the previous meta-analyses, our study has several strengths. We conducted subgroup meta-analyses by important factors that affect individual results and found out that the preventive effect of vitamin D supplements on ARIs was associated with the quality of the studies. In the subgroup meta-analysis, a significant preventive effect of vitamin D supplementation on ARIS was observed in daily supplementation and in the use of supplements during the short-term period. However, such beneficial effects disappeared in the subgroup meta-analysis of high-quality studies. That is, we think that the inconsistent findings of the previous meta-analyses might be associated with the study quality. Moreover, we used both the Jadad scale and Cochrane risk of bias tool to assess the methodological quality of the trials. Because the Jadad scale, which is a simple tool for 
assessing study quality, has been criticized by its generic problems of scale, we also used the Cochrane risk of bias tool for accuracy.

There are some limitations in this study. First, it would be ideal to investigate the efficacy of vitamin D supplementation on ARIs considering the baseline concentration of the $25(\mathrm{OH}) \mathrm{D}$. However, this was unavailable in most of the studies included in our analysis. Thus, we could not investigate the differences in the preventive effect on ARIs between people with vitamin D deficiency and normal vitamin D levels. Further clinical trials with the data of baseline $25(\mathrm{OH}) \mathrm{D}$ levels are warranted to confirm our findings. Second, publication bias was found in this study, which means that trials showing an increasing risk of or no effect on ARIs by vitamin D supplementation might not be published. This favors our conclusion that there is no preventive effect of vitamin D supplements on ARIs. Finally, several RCTs included in the current study were not designed specifically to investigate the efficacy of vitamin D supplements on ARIs as a primary endpoint. Findings in the secondary endpoint might be due to chance.

\section{Conclusions}

The current meta-analysis of RCTs shows that the use of vitamin D supplements has no efficacy in the prevention of ARIs.

Author Contributions: S.-K.M. has full access to all of the data in the study and takes responsibility for the integrity of the data and the accuracy of the data analysis. Conceptualization, S.-K.M.; literature search and assessment of study eligibility, H.-E.C. and H.C.; assessment of methodological quality, H.-E.C. and H.C.; statistical analysis and writing-original draft preparation, H.-E.C.; writing-review and editing, S.-K.M. and H.-E.C. All authors read and agreed to the published version of the manuscript.

Funding: This research received no external funding.

Institutional Review Board Statement: Not applicable.

Informed Consent Statement: Not applicable.

Data Availability Statement: The authors used published data from the individual studies and declare that the data supporting the findings of this study are available within the article.

Conflicts of Interest: All authors declare no conflict of interest.

\section{References}

1. Grief, S.N. Upper respiratory infections. Prim. Care Clin. Off. Pract. 2013, 40, 757-770. [CrossRef] [PubMed]

2. Barrett, B.T.; Goldman, R.B. Chapter 337: The common cold. In Cecil Medicine, 26th ed.; Goldman, L., Schafer, A.I., Eds.; Elsevier: Amsterdam, The Netherlands, 2019; pp. 2150-2152.

3. Musher, D.M. Chapter 91: Overview of pneumonia. In Cecil Medicine, 26th ed.; Goldman, L., Schafer, A.I., Eds.; Elsevier: Amsterdam, The Netherlands, 2019; pp. 592-603.

4. Troeger, C.; Forouzanfar, M.; Rao, P.C.; Khalil, I.; Brown, A.; Swartz, S.; Fullman, N.; Mosser, J.; Thompson, R.L.; Reiner, R.C., Jr.; et al. Estimates of the global, regional, and national morbidity, mortality, and aetiologies of lower respiratory tract infections in 195 countries: A systematic analysis for the Global Burden of Disease Study 2015. Lancet Infect. Dis. 2017, 17, 1133-1161. [CrossRef]

5. Mousa, H.A. Prevention and Treatment of Influenza, Influenza-Like Illness, and Common Cold by Herbal, Complementary, and Natural Therapies. J. Evid. Based Complementary Altern. Med. 2017, 22, 166-174. [CrossRef] [PubMed]

6. Abioye, A.I.; Bromage, S.; Fawzi, W. Effect of micronutrient supplements on influenza and other respiratory tract infections among adults: A systematic review and meta-analysis. BMJ Glob. Health 2021, 6, e003176. [CrossRef] [PubMed]

7. Sinopoli, A.; Isonne, C.; Santoro, M.M.; Baccolini, V. The effects of orally administered lactoferrin in the prevention and management of viral infections: A systematic review. Rev. Med. Virol. 2022, 32, e2261. [CrossRef] [PubMed]

8. Khammissa, R.A.G.; Fourie, J.; Motswaledi, M.H.; Ballyram, R.; Lemmer, J.; Feller, L. The Biological Activities of Vitamin D and Its Receptor in Relation to Calcium and Bone Homeostasis, Cancer, Immune and Cardiovascular Systems, Skin Biology, and Oral Health. BioMed Res. Int. 2018, 2018, 9276380. [CrossRef]

9. Cantorna, M.T.; Snyder, L.; Lin, Y.D.; Yang, L. Vitamin D and 1,25(OH)2D regulation of T cells. Nutrients 2015, 7, 3011-3021. [CrossRef]

10. Christakos, S.; Hewison, M.; Gardner, D.G.; Wagner, C.L.; Sergeev, I.N.; Rutten, E.; Pittas, A.G.; Boland, R.; Ferrucci, L.; Bikle, D.D. Vitamin D: Beyond bone. Ann. N. Y. Acad. Sci. 2013, 1287, 45-58. [CrossRef]

11. Hayashi, H.; Okamatsu, M.; Ogasawara, H.; Tsugawa, N.; Isoda, N.; Matsuno, K.; Sakoda, Y. Oral Supplementation of the Vitamin D Metabolite 25(OH)D3 Against Influenza Virus Infection in Mice. Nutrients 2020, 12, 2000. [CrossRef] 
12. Adenote, A.; Dumic, I.; Madrid, C.; Barusya, C.; Nordstrom, C.W.; Rueda Prada, L. NAFLD and Infection, a Nuanced Relationship. Can. J. Gastroenterol. Hepatol. 2021, 2021, 5556354. [CrossRef]

13. Laaksi, I.; Ruohola, J.P.; Mattila, V.; Auvinen, A.; Ylikomi, T.; Pihlajamaki, H. Vitamin D supplementation for the prevention of acute respiratory tract infection: A randomized, double-blinded trial among young Finnish men. J. Infect. Dis. 2010, 202, 809-814. [CrossRef] [PubMed]

14. Urashima, M.; Segawa, T.; Okazaki, M.; Kurihara, M.; Wada, Y.; Ida, H. Randomized trial of vitamin D supplementation to prevent seasonal influenza A in schoolchildren. Am. J. Clin. Nutr. 2010, 91, 1255-1260. [CrossRef] [PubMed]

15. Camargo, C.A., Jr.; Ganmaa, D.; Frazier, A.L.; Kirchberg, F.F.; Stuart, J.J.; Kleinman, K.; Sumberzul, N.; Rich-Edwards, J.W Randomized trial of vitamin D supplementation and risk of acute respiratory infection in Mongolia. Pediatrics 2012, 130, 561-567. [CrossRef] [PubMed]

16. Goodall, E.C.; Granados, A.C.; Luinstra, K.; Pullenayegum, E.; Coleman, B.L.; Loeb, M.; Smieja, M. Vitamin D3 and gargling for the prevention of upper respiratory tract infections: A randomized controlled trial. BMC Infect. Dis. 2014, 273, 1471-2334. [CrossRef]

17. Slow, S.; Priest, P.; Chambers, S.; Stewart, A.; Jennings, L.; Florkowski, C.; Livesey, J.; Camargo, C.; Scragg, R.; Murdoch, D. Effect of vitamin D3 supplementation on Staphylococcus aureus nasal carriage: A randomized, double-blind, placebo-controlled trial in healthy adults. Clin. Microbiol. Infect. 2014, 20, 453-458. [CrossRef]

18. Bergman, P.; Norlin, A.C.; Hansen, S.; Bjorkhem-Bergman, L. Vitamin D supplementation to patients with frequent respiratory tract infections: A post hoc analysis of a randomized and placebo-controlled trial. BMC Res. Notes 2015, 8, 391. [CrossRef]

19. Ginde, A.A.; Blatchford, P.; Breese, K.; Zarrabi, L.; Linnebur, S.A.; Wallace, J.I.; Schwartz, R.S. High-Dose Monthly Vitamin D for Prevention of Acute Respiratory Infection in Older Long-Term Care Residents: A Randomized Clinical Trial. J. Am. Geriatr. Soc. 2017, 65, 496-503. [CrossRef]

20. Hibbs, A.M.; Ross, K.; Kerns, L.A.; Wagner, C.; Fuloria, M.; Groh-Wargo, S.; Zimmerman, T.; Minich, N.; Tatsuoka, C. Effect of Vitamin D Supplementation on Recurrent Wheezing in Black Infants Who Were Born Preterm: The D-Wheeze Randomized Clinical Trial. JAMA 2018, 319, 2086-2094. [CrossRef]

21. Shimizu, Y.; Ito, Y.; Yui, K.; Egawa, K.; Orimo, H. Intake of 25-Hydroxyvitamin D3 Reduces Duration and Severity of Upper Respiratory Tract Infection: A Randomized, Double-Blind, Placebo-Controlled, Parallel Group Comparison Study. J. Nutr. Health Aging 2018, 22, 491-500. [CrossRef]

22. Zhou, J.; Du, J.; Huang, L.; Wang, Y.; Shi, Y.; Lin, H. Preventive Effects of Vitamin D on Seasonal Influenza A in Infants: A Multicenter, Randomized, Open, Controlled Clinical Trial. Pediatr. Infect. Dis. J. 2018, 37, 749-754. [CrossRef]

23. Arihiro, S.; Nakashima, A.; Matsuoka, M.; Suto, S.; Uchiyama, K.; Kato, T.; Mitobe, J.; Komoike, N.; Itagaki, M.; Miyakawa, Y.; et al. Randomized Trial of Vitamin D Supplementation to Prevent Seasonal Influenza and Upper Respiratory Infection in Patients with Inflammatory Bowel Disease. Inflamm. Bowel Dis. 2019, 25, 1088-1095. [CrossRef]

24. Li-Ng, M.; Aloia, J.F.; Pollack, S.; Cunha, B.A.; Mikhail, M.; Yeh, J.; Berbari, N. A randomized controlled trial of vitamin D3 supplementation for the prevention of symptomatic upper respiratory tract infections. Epidemiol. Infect. 2009, 137, 1396-1404. [CrossRef] [PubMed]

25. Manaseki-Holland, S.; Maroof, Z.; Bruce, J.; Mughal, M.Z.; Masher, M.I.; Bhutta, Z.A.; Walraven, G.; Chandramohan, D. Effect on the incidence of pneumonia of vitamin D supplementation by quarterly bolus dose to infants in Kabul: A randomised controlled superiority trial. Lancet 2012, 379, 1419-1427. [CrossRef]

26. Murdoch, D.R.; Slow, S.; Chambers, S.T.; Jennings, L.C.; Stewart, A.W.; Priest, P.C.; Florkowski, C.M.; Livesey, J.H.; Camargo, C.A.; Scragg, R. Effect of Vitamin D3 Supplementation on Upper Respiratory Tract Infections in Healthy Adults: The VIDARIS Randomized Controlled Trial. JAMA 2012, 308, 1333-1339. [CrossRef] [PubMed]

27. Rees, J.R.; Hendricks, K.; Barry, E.L.; Peacock, J.L.; Mott, L.A.; Sandler, R.S.; Bresalier, R.S.; Goodman, M.; Bostick, R.M.; Baron, J.A. Vitamin D3 supplementation and upper respiratory tract infections in a randomized, controlled trial. Clin. Infect. Dis. 2013, 57, 1384-1392. [CrossRef] [PubMed]

28. Urashima, M.; Mezawa, H.; Noya, M.; Camargo, C.A., Jr. Effects of vitamin D supplements on influenza A illness during the 2009 H1N1 pandemic: A randomized controlled trial. Food Funct. 2014, 5, 2365-2370. [CrossRef] [PubMed]

29. Martineau, A.R.; Hanifa, Y.; Witt, K.D.; Barnes, N.C.; Hooper, R.; Patel, M.; Stevens, N.; Enayat, Z.; Balayah, Z.; Syed, A.; et al. Double-blind randomised controlled trial of vitamin D3 supplementation for the prevention of acute respiratory infection in older adults and their carers (ViDiFlu). Thorax 2015, 70, 953-960. [CrossRef]

30. Martineau, A.R.; James, W.Y.; Hooper, R.; Barnes, N.C.; Jolliffe, D.A.; Greiller, C.L.; Islam, K.; McLaughlin, D.; Bhowmik, A.; Timms, P.; et al. Vitamin D 3 supplementation in patients with chronic obstructive pulmonary disease (ViDiCO): A multicentre, double-blind, randomised controlled trial. Lancet Respir. Med. 2015, 3, 120-130. [CrossRef]

31. Martineau, A.R.; MacLaughlin, B.D.; Hooper, R.L.; Barnes, N.C.; Jolliffe, D.A.; Greiller, C.L.; Kilpin, K.; McLaughlin, D.; Fletcher, G.; Mein, C.A.; et al. Double-blind randomised placebo-controlled trial of bolus-dose vitamin D3 supplementation in adults with asthma (ViDiAs). Thorax 2015, 70, 451-457. [CrossRef]

32. Mayan, I.; Somech, R.; Lev, A.; Cohen, A.H.; Constantini, N.W.; Dubnov-Raz, G. Thymus Activity, Vitamin D, and Respiratory Infections in Adolescent Swimmers. Isr. Med. Assoc. J. 2015, 17, 571-575. 
33. Denlinger, L.C.; King, T.S.; Cardet, J.C.; Craig, T.; Holguin, F.; Jackson, D.J.; Kraft, M.; Peters, S.P.; Ross, K.; Sumino, K.; et al. Vitamin D Supplementation and the Risk of Colds in Patients with Asthma. Am. J. Respir. Crit. Care Med. 2016, 193, 634-641. [CrossRef] [PubMed]

34. Gupta, P.; Dewan, P.; Shah, D.; Sharma, N.; Bedi, N.; Kaur, I.R.; Bansal, A.K.; Madhu, S.V. Vitamin D Supplementation for Treatment and Prevention of Pneumonia in Under-five Children: A Randomized Double-blind Placebo Controlled Trial. Indian Pediatr. 2016, 53, 967-976. [CrossRef]

35. Aglipay, M.; Birken, C.S.; Parkin, P.C.; Loeb, M.B.; Thorpe, K.; Chen, Y.; Laupacis, A.; Mamdani, M.; MacArthur, C.; Hoch, J.S.; et al Effect of High-Dose vs Standard-Dose Wintertime Vitamin D Supplementation on Viral Upper Respiratory Tract Infections in Young Healthy Children. JAMA 2017, 318, 245-254. [CrossRef] [PubMed]

36. Brett, N.R.; Lavery, P.; Agellon, S.; Vanstone, C.A.; Goruk, S.; Field, C.J.; Weiler, H.A. Vitamin D Status and Immune Health Outcomes in a Cross-Sectional Study and a Randomized Trial of Healthy Young Children. Nutrients 2018, 10, 680. [CrossRef]

37. Loeb, M.; Dang, A.D.; Thiem, V.D.; Thanabalan, V.; Wang, B.; Nguyen, N.B.; Tran, H.T.M.; Luong, T.M.; Singh, P.; Smieja, M.; et al Effect of Vitamin D supplementation to reduce respiratory infections in children and adolescents in Vietnam: A randomized controlled trial. Influenza Other Respir. Viruses 2019, 13, 176-183. [CrossRef] [PubMed]

38. Singh, N.; Kamble, D.; Mahantshetti, N.S. Effect of Vitamin D Supplementation in the Prevention of Recurrent Pneumonia in Under-Five Children. Indian J. Pediatr. 2019, 86, 1105-1111. [CrossRef] [PubMed]

39. Camargo, C.A.; Sluyter, J.; Stewart, A.W.; Khaw, K.T.; Lawes, C.M.; Toop, L.; Waayer, D.; Scragg, R. Effect of Monthly High-Dose Vitamin D Supplementation on Acute Respiratory Infections in Older Adults: A Randomized Controlled Trial. Clin. Infect. Dis. 2020, 71, 311-317. [CrossRef]

40. Ganmaa, D.; Uyanga, B.; Zhou, X.; Gantsetseg, G.; Delgerekh, B.; Enkhmaa, D.; Khulan, D.; Ariunzaya, S.; Sumiya, E.; Bolortuya, B.; et al. Vitamin D Supplements for Prevention of Tuberculosis Infection and Disease. N. Engl. J. Med. 2020, 383, 359-368. [CrossRef]

41. Sudfeld, C.R.; Mugusi, F.; Muhihi, A.; Aboud, S.; Nagu, T.J.; Ulenga, N.; Hong, B.; Wang, M.; Fawzi, W.W. Efficacy of vitamin D3 supplementation for the prevention of pulmonary tuberculosis and mortality in HIV: A randomised, double-blind, placebocontrolled trial. Lancet HIV 2020, 7, 463-471. [CrossRef]

42. Pham, H.; Waterhouse, M.; Baxter, C.; Romero, B.D.; McLeod, D.S.; Armstrong, B.K.; Ebeling, P.R.; English, D.R.; Hartel, G.; Kimlin, M.G.; et al. The effect of vitamin D supplementation on acute respiratory tract infection in older Australian adults: An analysis of data from the D-Health Trial. Lancet Diabetes Endocrinol. 2021, 9, 69-81. [CrossRef]

43. Xiao, L.; Xing, C.; Yang, Z.; Xu, S.; Wang, M.; Du, H.; Liu, K.; Huang, Z. Vitamin D supplementation for the prevention of childhood acute respiratory infections: A systematic review of randomised controlled trials. Br. J. Nutr. 2015, 114, 1026-1034 [CrossRef] [PubMed]

44. Martineau, A.R.; Jolliffe, D.A.; Greenberg, L.; Aloia, J.F.; Bergman, P.; Dubnov-Raz, G.; Esposito, S.; Ganmaa, D.; Ginde, A.A.; Goodall, E.C.; et al. Vitamin D supplementation to prevent acute respiratory infections: Individual participant data meta-analysis. Health Technol. Assess. 2019, 23, 1-44. [CrossRef] [PubMed]

45. Pham, H.; Rahman, A.; Majidi, A.; Waterhouse, M.; Neale, R.E. Acute Respiratory Tract Infection and 25-Hydroxyvitamin D Concentration: A Systematic Review and Meta-Analysis. Int. J. Environ. Res. Public Health 2019, 16, 3020. [CrossRef]

46. Jolliffe, D.A.; Camargo, C.A., Jr.; Sluyter, J.D.; Aglipay, M.; Aloia, J.F.; Ganmaa, D.; Bergman, P.; Bischoff-Ferrari, H.A.; Borzutzky, A.; Damsgaard, C.T. Vitamin D supplementation to prevent acute respiratory infections: A systematic review and meta-analysis of aggregate data from randomised controlled trials. Lancet Diabetes Endocrinol. 2021, 9, 276-292. [CrossRef]

47. Jadad, A.R.; Moore, R.A.; Carroll, D.; Jenkinson, C.; Reynolds, D.J.; Gavaghan, D.J. Assessing the quality of reports of randomized clinical trials: Is blinding necessary? Control. Clin. Trials. 1996, 17, 1-12. [CrossRef]

48. Higgins, J.P.T.; Savovic, J.; Page, M.J.; Elbers, R.G.; Sterne, J.A.C. Assessing risk of bias in a randomized trial. In Cochrane Handbook for Systematic Reviews of Interventions; Higgins, J.P.T., Thomas, J., Chandler, J., Cumpston, M., Li, T., Page, M.J., Welch, V.A., Eds.; Wiley-Blackwell: Chichester, UK, 2020.

49. Higgins, J.P.; Thompson, S.G. Quantifying heterogeneity in a meta-analysis. Stat. Med. 2002, 21, 1539-1558. [CrossRef] [PubMed]

50. Reid, I.R.; Ames, R.W.; Evans, M.C.; Gamble, G.D.; Sharpe, S.J. Long-term effects of calcium supplementation on bone loss and fractures in postmenopausal women: A randomized controlled trial. Am. J. Med. 1995, 98, 331-335. [CrossRef]

51. Beard, J.A.; Bearden, A.; Striker, R. Vitamin D and the anti-viral state. J. Clin. Virol. 2011, 50, 194-200. [CrossRef]

52. Wei, R.; Christakos, S. Mechanisms Underlying the Regulation of Innate and Adaptive Immunity by Vitamin D. Nutrients 2015, 7, 8251-8260. [CrossRef]

53. White, J.H. Emerging Roles of Vitamin D-Induced Antimicrobial Peptides in Antiviral Innate Immunity. Nutrients 2022, 14, 284. [CrossRef] 